\title{
Controlling the Degree of Esterification of Citrus Pectin for Demanding Applications by Selection of the Source
}

\author{
Rosaria Ciriminna, ${ }^{\dagger}$ Alexandra Fidalgo, ${ }^{\ddagger}$ Riccardo Delisi, ${ }^{\dagger}$ Alfredo Tamburino, ${ }^{\perp}$ Diego Carnaroglio, ${ }^{\S}, \|$

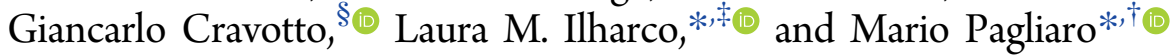 \\ ${ }^{\dagger}$ Istituto per lo Studio dei Materiali Nanostrutturati, CNR, via U. La Malfa 153, 90146 Palermo, Italy \\ *Centro de Química-Física Molecular and IN-Institute of Nanoscience and Nanotechnology, Instituto Superior Técnico, Universidade \\ de Lisboa, Av. Rovisco Pais 1, 1049-001 Lisboa, Portugal \\ ${ }^{\S}$ Dipartimento di Scienza e Tecnologia del Farmaco and Centre for Nanostructured Interfaces and Surfaces, Università degli Studi di \\ Torino, via P. Giuria 9, 10125 Torino, Italy \\ "Milestone, via Fabenefratelli, 1-5, 24010 Sorisole, Bergamo, Italy \\ ${ }^{\perp}$ Dipartimento Agricoltura, Legambiente Sicilia, via Tripoli 3, 90138 Palermo, Italy
}

\section{Supporting Information}

ABSTRACT: Analyzed by a quantitative method based on diffuse reflectance infrared Fourier transform spectroscopy, pectins extracted from different regions (outer skin, peel, and waste) of citrus fruits (red orange, lemon, and grapefruit) via microwave-assisted hydrodiffusion show significant variations. All polymers obtained are low-methoxyl pectins, with high contents in galacturonic acid regions. The degree of esterification (DE) of pectin extracted from different regions increases in the order waste $<$ peel $<$ outer skin for red orange, inverting for lemon. Thus, the pectins with the lowest DE are those extracted from red orange waste and lemon outer skin ( 25\%). These findings open the route to nutraceutical- and pharmaceutical-grade pectins from citrus, in which the source fruit and its regions may be chosen, according to the desired DE.

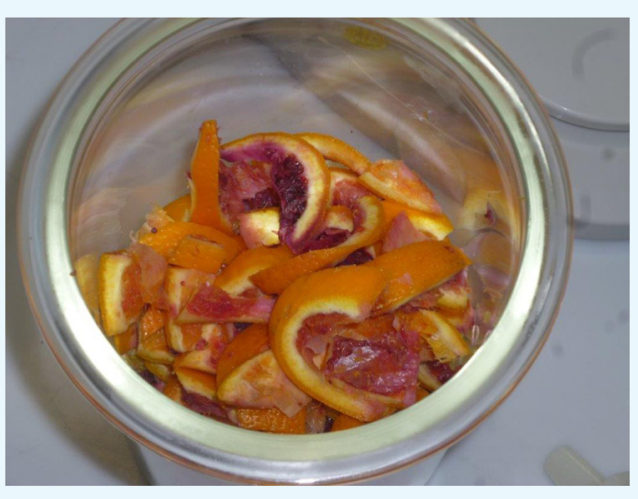

\section{INTRODUCTION}

Industrially obtained from lemon peel and, to a minor extent, from apple pomace, via acid hydrolysis using aqueous hydrochloric acid, nitric acid, or sulfuric acid at high temperature under controlled conditions $(\mathrm{pH}$, time, and temperature) to minimize deesterification and depolymerization, ${ }^{1}$ heteropolysaccharide pectin is a natural hydrocolloid currently found in multiple food, cosmetic, beverage, pharmaceutical, and nutraceutical products, in which it exerts important functions, including gelation, fiber enrichment, stabilization, and texture and rheology control. ${ }^{2}$ The chemical structure of pectin depends on the source, location in the source, and extraction method. In general, the structure is mostly composed of homopolymeric partially 6-methylated and 2 - and/or 3-acetylated poly- $\alpha(1-4)$-D-galacturonic acid residues (known as homogalacturonan, HG, regions), alternating with branched $\alpha(1-2)$-L-rhamnosyl- $\alpha(1-4)$-D-galacturonosyl chains substituted with side chains of mainly $\alpha$-L-arabinofuranose and $\alpha$-D-galactopyranose (known as rhamnogalacturonan I, RG-I, regions). ${ }^{3}$ The relative proportions of interconnected HG and RG-I regions, also called "smooth" (HG) and "hairy" (RG-I) regions, respectively, determine the flexibility and rheological properties of the polymer in solution: the HG regions enhance molecular interactions, whereas the branched
$\mathrm{RG}$ regions promote the formation of entangled structures. ${ }^{4} \mathrm{On}$ the other hand, some of the carboxyl groups in the galacturonic acid residues, both in the HG and RG regions, may be methylesterified or exist as uronic acid salt, affecting the polymer charge in solution. ${ }^{5}$

Such structural features turn pectin into an extremely versatile polymer. With high molecular weights (80-400 $\mathrm{kDa}$ ), pectins are easily converted into hydrogels (HG domains are responsible for the gelling capability, while RG-I regions play a gel-stabilizing role), whose gelling mechanism is dictated by their degree of esterification (DE, total methoxyl content): pectins with high $\mathrm{DE}$ tend to form gels at low $\mathrm{pH}$, stabilized by hydrophobic interactions, ${ }^{6}$ whereas those with low DE tend to form gels electrostatically stabilized by metal cations. ${ }^{5}$ This gelling behavior shows how important it is to consider simultaneously the DE, the monosaccharide content (HG), and the spatial disposition of the cross-linking blocks (RG) when designing pectin gels for specific applications.?

Commercially, pectins are classified according to their methoxyl content and to the gel-forming rate. In detail, pectin

Received: August 1, 2017

Accepted: November 3, 2017

Published: November 16, 2017 
commercial grades with DE lower and higher than 50\% are classified as low and high methoxyl (LM and HM) pectins, respectively. Due to booming demand facing saturated capacity of existing extraction plants relying on conventional extraction process, the price of both LM and HM pectins has more than doubled. ${ }^{8}$ Furthermore, pectin is being increasingly demanded for nutraceutical applications due to numerous health benefits, including its ability to inhibit galectin- $3^{10}$ and to lower cholesterol and glucose levels in blood, for which the European Food Safety Authority recommends intake of $6 \mathrm{~g}$ of pectin per day. ${ }^{11}$ Indeed, pectin's source, degree of esterification, and molecular weight crucially affect its ability to lower cholesterol levels in blood, leading scholars to conclude that authorities issuing pectin cholesterol-lowering claim should require inclusion of $\mathrm{DE}$ and molecular weight. ${ }^{12}$

Intensive research efforts are being devoted across the world to develop new commercial pectin sources and extraction techniques. ${ }^{13}$ In this context, we have lately shown how to extract citrus pectin and essential oils from waste orange and lemon peel in high yield by an ecofriendly process run entirely in water, combining solvent-free microwave extraction and microwave hydrodiffusion and gravity. ${ }^{14}$ Opposite to what happens in a conventional hydrodistillation, during microwave extraction, the heating of the biological matrix takes place outwardly, generally affording high extraction yields at lower temperatures, which makes the process well suited for valorization of fruit and agriculture coproducts in terms of energy, elimination of solvents, and enhanced yield. ${ }^{15}$ In 2013, for example, scholars in India reported that optimized microwave-assisted extraction of pectin from dried orange peel in water at $\mathrm{pH} 1.4$ afforded a $19.24 \%$ pectin yield. ${ }^{16}$ Excellent results with waste orange peel microwave extraction have been reported by Clark's team already in $2012 .{ }^{17}$

We now report the results of the extraction of pectin from different (undried) citrus fruits and from different fruit parts, namely, the outer skin (exocarp), the peel (exo- and mesocarp), and the waste (exo-, meso-, and endocarp) of Sicily's red orange and lemon fruits, as well as the peel of grapefruit, all collected between December and early January (i.e., the best picking period of the year), using microwave-assisted hydrodiffusion. ${ }^{18}$ The pectins are analyzed by infrared spectroscopy (in diffuse reflectance mode), leading to differentiate them in terms of degree of esterification and relative content in smooth regions. This work represents a proof of concept of the following: pectin with the desired degree of esterification may be obtained just by a judicious choice of the source (fruit and/ or fruit part), the degree of esterification being determined by a simple, experimentally straightforward, nondestructive, and time-saving method (quantitative analysis of diffuse reflectance infrared Fourier transform (DRIFT) spectra).

\section{RESULTS AND DISCUSSION}

Different fresh citrus fruits and fruit parts were subjected to microwave extraction in water using a laboratory-scale commercial microwave extractor (see Experimental Section). After irradiating each sample at $600 \mathrm{~W}$ until the temperature reached $100{ }^{\circ} \mathrm{C}$, the power was lowered to $500 \mathrm{~W}$. The total time of irradiation lasted between 60 and $80 \mathrm{~min}$ (Table 1), depending on the amount of each sample as well as on its water content.

The $20 \mathrm{~min}$ difference between the shortest and longest irradiation times only depends on the time needed for optimal extraction under $500 \mathrm{~W}$ microwave irradiation. After a certain
Table 1. Extraction Time and Weight of Fresh Citrus Source Subjected to Microwave Extraction in Water

\begin{tabular}{lcc}
\multicolumn{1}{c}{ pectin source } & time $(\mathrm{min})$ & fresh weight $(\mathrm{g})$ \\
red orange outer skin & 75 & 445 \\
red orange peel & 60 & 500 \\
red orange waste & 80 & 500 \\
lemon outer skin & 60 & 500 \\
lemon peel & 80 & 500 \\
lemon waste & 70 & 500 \\
grapefruit peel & 70 & 500
\end{tabular}

time, indeed, the matrix sample being extracted suspended in hot water tends to turn brown due to deep dehydration. A typical water extract obtained from waste red orange peel, with the insoluble essential oil easily decanted, is shown in Figure $1 .{ }^{19}$

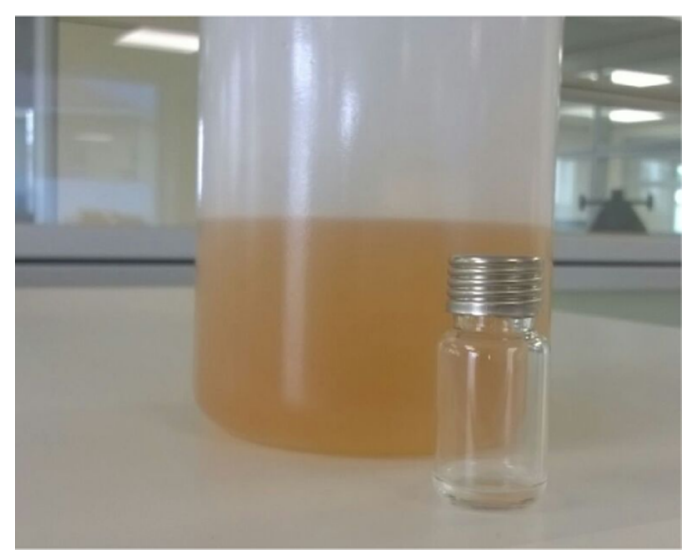

Figure 1. Water extract (on the background) and essential oil (in the foreground) obtained from waste red orange peel via microwaveassisted extraction in water. Photograph courtesy of Dr. D. Carnaroglio. Copyright 2017.

All samples were extensively characterized by DRIFT spectroscopy (Figure 2). The complete assignments are included in Table S1 of the Supporting information (SI). Given that the spectra are normalized to the $\nu \mathrm{C}-\mathrm{O}-\mathrm{C}$ band of pyranose rings (at $\sim 1100 \mathrm{~cm}^{-1}$ ), the relative intensities of the carboxylate-related bands change very clearly, especially for pectin extracted from lemon, suggesting different degrees of esterification.

The influence of the type of fruit on the spectrum of pectin extracted from equivalent regions is well expressed in Figure 3.

Although the spectra of pectin from the exocarp (outer skin) of the different fruits are similar, those of pectin from the waste apparently show different degrees of esterification, and those from the peel (exo- and mesocarp) are completely different, suggesting also different contents of galacturonic acid-rich (HG) regions.

The DE of pectin has been determined by Monsoor et al. from the DRIFT spectra, by correlating the ratio of ester carboxyl to total carboxyl peak areas with the values obtained by gas chromatography. ${ }^{20}$ We have recently used a similar approach consisting in determining $\mathrm{DE}$ directly from the ratio of band intensities of the esterified carboxyl stretching components to the total band intensities of the esterified plus nonesterified carboxylate groups, obtained by spectral deconvolution. ${ }^{14}$ A detailed spectral analysis was necessary. It implied 

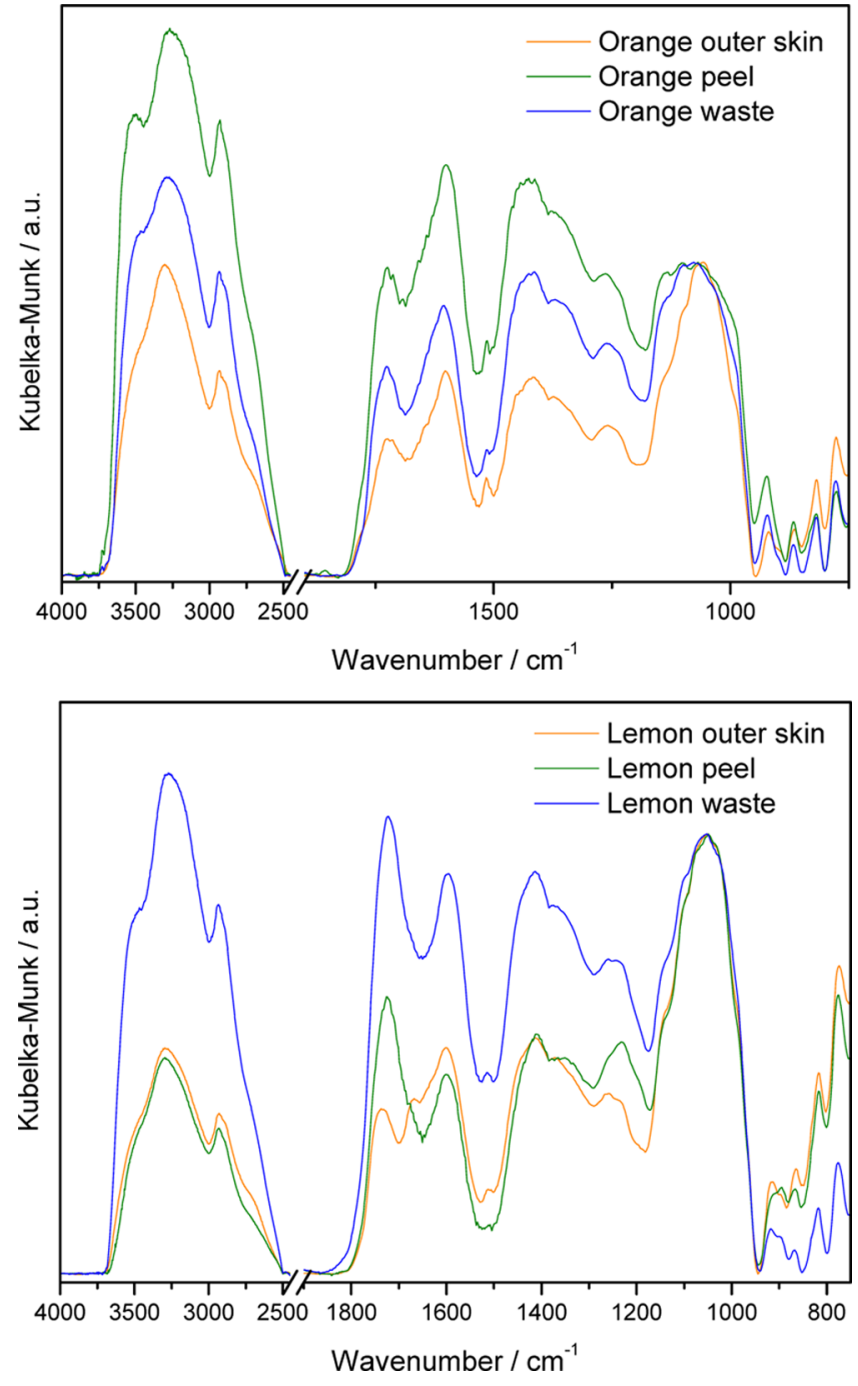

Figure 2. DRIFT spectra of pectin from different parts of the same fruit, as indicated. The spectra are normalized to the maximum of the pyranose $\nu \mathrm{C}-\mathrm{O}-\mathrm{C}$ band, at $\sim 1100 \mathrm{~cm}^{-1}$.

the assignment of vibrational components not directly observed in the region related to the carbonyl and carboxylate stretching modes $\left(1800-1500 \mathrm{~cm}^{-1}\right)$ : because the carbonyl groups may be involved in different hydrogen bonds and therefore contribute with more than one component, the best estimates for the $\nu \mathrm{C}=\mathrm{O}$ and $\nu_{\mathrm{as}} \mathrm{COO}^{-}$band areas were obtained by decomposing the whole $1900-900 \mathrm{~cm}^{-1}$ region into a sum of Gaussian and Lorentzian components, ${ }^{21}$ using a nonlinear leastsquares fitting method. The detailed results of the deconvolutions are included in Table S2 of the Supporting information (SI).

The DE values were thus obtained by eq $1^{14}$

$$
\begin{aligned}
\mathrm{DE}= & \sum A_{\nu(\mathrm{C}=\mathrm{O}) \text { ester }} /\left(\sum A_{\nu(\mathrm{C}=\mathrm{O}) \text { ester }}+A_{\nu(\mathrm{C}=\mathrm{O}) \text { acid }}\right. \\
& \left.+A_{\nu \mathrm{asCOO}^{-}}\right)
\end{aligned}
$$

As to the amount of galacturonic acid-rich (HG) regions of pectin, their values are not calculated, but they are proportional to the ratio described by eq $2,{ }^{14}$ also assessed from the quantitative analysis of the DRIFT spectra
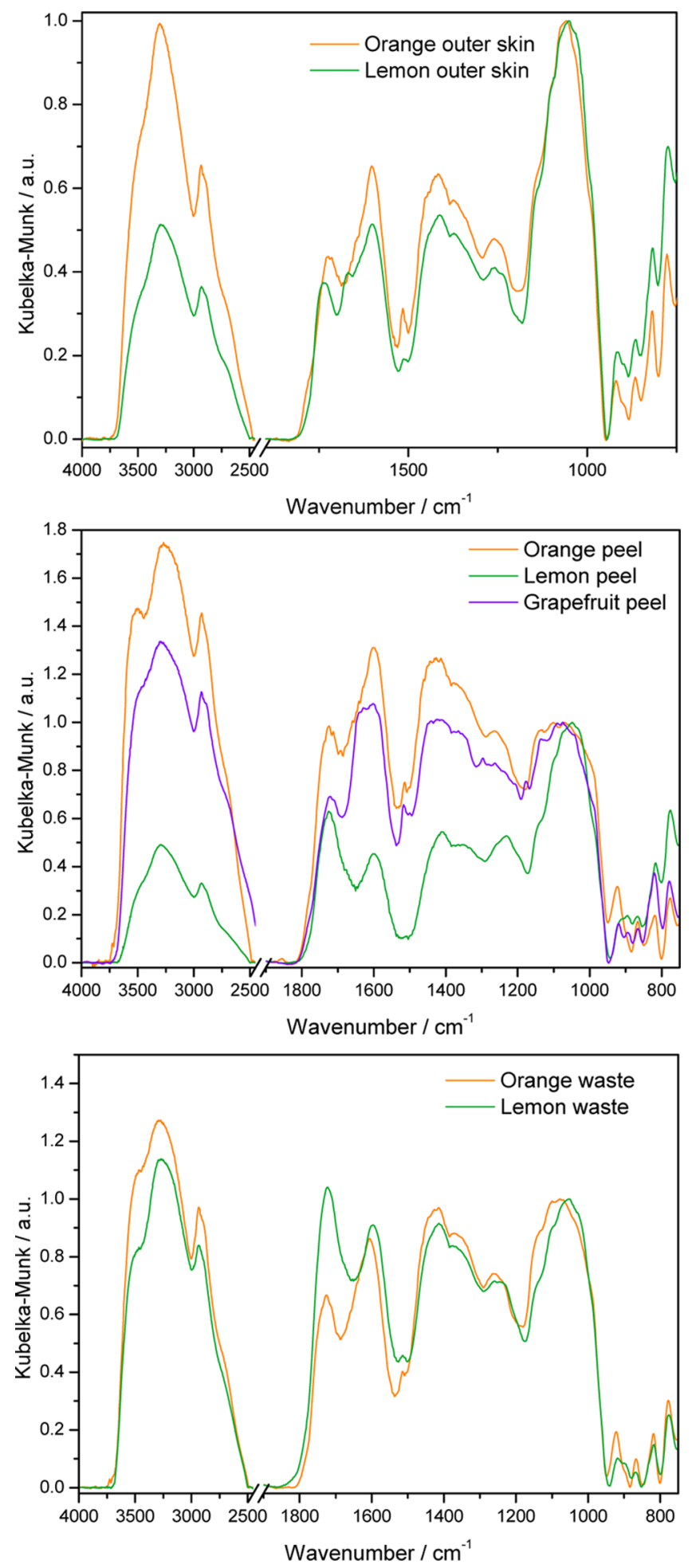

Figure 3. DRIFT spectra of pectin from equivalent regions of different fruits, as indicated.

$$
\begin{aligned}
\mathrm{HG} \propto & {\left[\sum A_{\nu(\mathrm{C}=\mathrm{O})}+A_{\nu \mathrm{asCOO}}\right] } \\
/ & {\left[\sum A_{\nu(\mathrm{C}-\mathrm{O}-\mathrm{C})}+\sum A_{\nu(\mathrm{C}=\mathrm{O})}+A_{\nu \mathrm{as} \mathrm{COO}^{-}}\right] }
\end{aligned}
$$

The values obtained are summarized in Table 2 .

In general, the results summarized in Table 2 show that pectin extracted from all of these sources falls into the low methoxyl category ( $\mathrm{DE}<50 \%$ ), namely, pectins of high quality 
Table 2. Degree of Esterification (DE) and Relative HG Content for Pectin Samples from Citrus Matrices Obtained via Hand-Cutting or Industrial Squeezing

\begin{tabular}{lcc}
\multicolumn{1}{c}{ pectin source } & DE $(\%)$ & HG $\propto$ to $(\%)$ \\
red orange outer skin & 39 & 43 \\
red orange peel & 30 & 58 \\
red orange waste & 25 & 48 \\
lemon outer skin & 24 & 37 \\
lemon peel & 34 & 42 \\
lemon waste & 40 & 58 \\
grapefruit peel & 34 & 55 \\
\hline
\end{tabular}

suitable as additives in the food industry, especially in low-fat products. However, the DE depends on the source, ranging from a minimum of $24 \%$ for pectin from lemon exocarp to a maximum of $40 \%$ for pectin extracted from lemon waste (exo-, meso-, and endocarp). The red orange (Citrus sinensis) and grapefruit pectins show intermediate values of DE. Besides, the $\mathrm{DE}$ increases from waste to outer skin in red orange pectin. This suggests that the pectin from the red orange pulp must have a very low DE (in contrast to the pectin of blond Valencia orange $)^{22}$ and a significant contribution.

In contrast, for lemon pectin, the minimum $\mathrm{DE}$ was obtained for the outer skin and the maximum for the waste. Interestingly, pectins from the peel, independently of the fruit, have similar degrees of esterification ( $30 \%)$. In summary, the fruit and its region may be chosen as a source of pectin according to the intended DE or, in other words, the pectin DE may be tuned by choosing the appropriate source.

The relative scale in HG content enables only to conclude that pectins from lemon waste, red orange peel, and grapefruit peel are the richest in galacturonic acid regions, whereas those from lemon outer skin are the poorest. In general, a higher percentage of HG regions in pectin from a certain fruit part can be explained by an increased breaking of RG backbone units upon squeezing, leading to a more aggregated structure. Apparently, the fruit pulps differ not only in the DE but also in the HG percentage. Pectin extracted from red orange waste combines low methoxyl content with high HG relative content.

Finally, confirmation of the results obtained by the present method was achieved by application to commercial pectin samples, whose degrees of esterification, provided by the manufacturers, nicely match those determined in this work (Table 3). The spectra are shown in Figure S1 of the SI, and the deconvolution results are detailed in Table S3 of the SI.

The minimum content in galacturonic acid regions was provided only for one commercial pectin $(74 \%$, for citrus pectin B). This is an indication that all of the pectin samples extracted in this work contain very high proportions of HG regions, which is a consequence of the low molecular degradation

Table 3. Degree of Esterification of Commercial Pectin Samples, According to Label and This Work

\begin{tabular}{ccccc} 
& \multicolumn{2}{c}{$\mathrm{DE}(\%)$} & & $\mathrm{HG} \propto$ to $(\%)$ \\
\cline { 2 - 3 } \cline { 5 - 5 } commercial sample & manufacturer & this work & & this work \\
citrus pectin A & $67-71$ & 68 & & 17 \\
citrus pectin B & & 62 & 44 \\
citrus pectin C & $55-70$ & 67 & & 35 \\
citrus pectin D & $>85$ & 86 & & 37
\end{tabular}

${ }^{a}$ Esterified potassium salt. ${ }^{b}$ Esterified. associated with the extraction procedure. Thus, the pectins extracted with microwaves only in neutral water from the Sicily citrus fruits in December/January appear to be generally richer in smooth regions than the commercial ones obtained via hydrolysis in hot water.

\section{CONCLUSIONS}

Pectins extracted with microwaves from outer skin, peel, and waste of red orange, lemon, and grapefruit harvested in Sicily are low-methoxyl pectins, in which the degree of esterification increases in the order waste $<$ peel < skin for red orange pectin, and inverts for lemon. The pectin extracted from lemon exocarp has not only a minimum degree of esterification (DE) but also a minimum content in smooth regions, whereas the pectin extracted from red orange waste (exo-, meso-, and endocarp) combines low methoxyl content with high HG content. The analytical method based on vibrational spectroscopy has been applied to samples of commercial citrus pectins. Remarkably, the results obtained with the new method are within the DE range reported by pectin manufacturers. These findings may open the route to high-quality nutraceutical- and pharmaceutical-grade citrus pectins obtained via an intrinsically clean process, employing pure water as the only extraction medium, in which the fruit and its region are chosen as a source of pectin according to the intended DE. We are currently studying and will shortly report the antioxidant and antimicrobial role imparted to these new pectins by the soluble flavonoid biophenols contained in the orange, ${ }^{23}$ lemon, ${ }^{24}$ and grapefruit $^{25}$ exocarp. It is also of relevance, in conclusion, that a similar versatility enabling to selectively adjust the essential oil composition has been recently reported also when microwave hydrodiffusion and gravity extraction were applied to waste lemon peel carried out on semi-industrial scale. ${ }^{26}$

\section{EXPERIMENTAL SECTION}

Pectins were extracted from undried citrus fruit parts of lemon, red orange, orange, and grapefruit using an NEOS microwave extractor (Milestone, Italy). Red orange and lemon wastes were obtained either by hand or by industrial squeezing (gift of Ortogel, Italy). The outer skins and peels of the three fruits were obtained in the laboratory by hand-cutting the fresh fruits. In a typical extraction, $500 \mathrm{~g}$ of citrus fruit parts and $400 \mathrm{~mL}$ of distilled water were added to a glass vessel. The vessel was inserted in the microwave oven of the NEOS commercial extractor. Its door was closed and power was set at $600 \mathrm{~W}$ until the temperature reached $100{ }^{\circ} \mathrm{C}$, after which power was lowered to $500 \mathrm{~W}$. The total time of irradiation lasted between 60 and $80 \mathrm{~min}$ (Table 1), depending on the amount of each sample as well as its water content. Before observing any browning, irradiation was switched off and the sample was allowed to cool down.

Pectin obtained via freeze drying as a pale orange or slightly yellow powder, respectively, from red orange or lemon and grapefruit matrices had a delicate and pleasant smell. The structure of said powders was assessed by Fourier transform infrared (FTIR) spectroscopy in diffuse reflectance mode (DRIFT), using a Mattson FTIR spectrometer with a Specac Selector, in the range of $4000-400 \mathrm{~cm}^{-1}$ (wide-band MCT detector), at $2 \mathrm{~cm}^{-1}$ resolution. The sample preparation consisted simply in grinding the sample and mixing with ground $\mathrm{KBr}$ in the appropriate proportion to assure the validity of the Kubelka-Munk assumptions. ${ }^{27}$ The spectra were the 
result of ratioing 1000 added scans for each sample against the same number of scans for the background (ground $\mathrm{KBr}$ ).

\section{ASSOCIATED CONTENT}

\section{S Supporting Information}

The Supporting Information is available free of charge on the ACS Publications website at DOI: 10.1021/acsomega.7b01109.

Assignment of the infrared spectra for all samples (Table S1) (PDF)

\section{AUTHOR INFORMATION}

\section{Corresponding Authors}

*E-mail: lilharco@tecnico.ulisboa.pt (L.M.I.).

*E-mail: mario.pagliaro@cnr.it (M.P.).

\section{ORCID}

Giancarlo Cravotto: 0000-0001-7574-7350

Laura M. Ilharco: 0000-0001-6994-1464

Mario Pagliaro: 0000-0002-5096-329X

Notes

The authors declare no competing financial interest.

\section{ACKNOWLEDGMENTS}

This article is dedicated to Prof. Cristina Della Pina, University of Milano, on the occasion of her recent appointment as Associate Professor of general chemistry.

\section{REFERENCES}

(1) Chan, S. Y.; Choo, W. S.; Young, D. J.; Loh, X. J. Pectin as a rheology modifier: Origin, structure, commercial production and rheology. Carbohydr. Polym. 2017, 161, 118-139.

(2) Ciriminna, R; Chavarría-Hernández, N.; Hernández, A. I. R.; Pagliaro, M. Pectin: A New Perspective from the Biorefinery Standpoint. Biofuels, Bioprod. Biorefin. 2015, 9, 368-377.

(3) Mohnen, D. Pectin structure and biosynthesis. Curr. Opin. Plant Biol. 2008, 11, 266-277.

(4) Morra, M.; Cassinelli, C.; Cascardo, G.; Nagel, M.-D.; Volpe, C. D.; Siboni, S.; Maniglio, D.; Brugnara, M.; Ceccone, G.; Schols, H. A.; Ulvskov, P. Effects on Interfacial Properties and Cell Adhesion of Surface Modification by Pectic Hairy Regions. Biomacromolecules 2004, 5, 2094-2104.

(5) Morris, G. A.; Foster, T. J.; Harding, S. E. The effect of the degree of esterification on the hydrodynamic properties of citrus pectin. Food Hydrocolloids 2000, 14, 227-235.

(6) Harding, S. E.; Tombs, M. P.; Adams, G. G.; Paulsen, B. S.; Inngjerdingen, K. T.; Barsett, H. An Introduction to Polysaccharide Biotechnology; CRC Press: Boca Raton, FL, 2017; pp 99-126.

(7) Ciriminna, R.; Fidalgo, A. M. A.; Delisi, R.; Ilharco, L. M.; Pagliaro, M. Pectin Production and Global Market. Agro Food Ind. Hi Tech 2016, 27, 17-20.

(8) In early $2007 \mathrm{LM}$ pectin was priced at $\$ 10.60$ per $\mathrm{lb}$, more than double its price in 2006 , and HM pectin at $\$ 9$ per lb. See: J. Gelski, Finding alternatives for vanilla, pectin, foodbusinessnews.net, June 3, 2017.

(9) Maxwell, E. G.; Belshaw, N. J.; Waldron, K. W.; Morris, V. J. Pectin - An emerging new bioactive food polysaccharide. Trends Food Sci. Technol. 2012, 24, 64-73.

(10) Kolatsi-Joannou, M.; Price, K. L.; Winyard, P. J.; Long, D. A. Modified citrus pectin reduces galectin-3 expression and disease severity in experimental acute kidney injury. PLoS One 2011, 6, No. e18683.

(11) EFSA Panel on Dietetic Products, Nutrition and AllergiesScientific Opinion on the substantiation of health claims related to pectins and reduction of post-prandial glycaemic responses (ID 786), maintenance of normal blood cholesterol concentrations (ID 818) and increase in satiety leading to a reduction in energy intake (ID 4692) pursuant to Article 13(1) of Regulation (EC) No 1924/2006. EFSA J. 2010, 8 1747. 10.2903/j.efsa.2010.1747.

(12) Brouns, F.; Theuwissen, E.; Adam, A.; Bell, M.; Berger, A.; Mensink, R. P. Cholesterol-lowering properties of different pectin types in mildly hyper-cholesterolemic men and women. Eur. J. Clin Nutr. 2012, 66, 591-599.

(13) Adetunji, L. R.; Adekunle, A.; Orsat, V.; Raghavan, V. Advances in the pectin production process using novel extraction techniques: $\mathrm{A}$ review. Food Hydrocolloids 2017, 62, 239-250.

(14) Fidalgo, A.; Ciriminna, R.; Carnaroglio, D.; Tamburino, A.; Cravotto, G.; Grillo, G.; Ilharco, L. M.; Pagliaro, M. Eco-Friendly Extraction of Pectin and Essential Oils from Orange and Lemon Peels. ACS Sustainable Chem. Eng. 2016, 4, 2243-2251.

(15) Jacotet-Navarro, M.; Rombaut, N.; Deslis, S.; Fabiano-Tixier, A.S.; Pierre, F.-X.; Bily, A.; Chemat, F. Towards a "dry" bio-refinery without solvents or added water using microwaves and ultrasound for total valorization of fruit and vegetable by-products. Green Chem. 2016, 18, 3106-3115.

(16) Maran, J. P.; Sivakumar, V.; Thirugnanasambandham, K.; Sridhar, R. Optimization of microwave assisted extraction of pectin from orange peel. Carbohydr. Polym. 2013, 97, 703-709.

(17) Balu, A. M.; Budarin, V.; Shuttleworth, P. S.; Pfaltzgraff, L. A.; Waldron, K.; Luque, R.; Clark, J. H. Valorisation of Orange Peel Residues: Waste to Biochemicals and Nanoporous Materials. ChemSusChem 2012, 5, 1694-1697.

(18) Vian, M. A.; Fernandez, X.; Visinoni, F.; Chemat, F. Microwave hydrodiffusion and gravity, a new technique for extraction of essential oils. J. Chromatogr. A 2008, 1190, 14-17.

(19) Marchese, D. Citrus Consumers Trend in Europe. New Tastes Sensation: The Blood Orange Juice Case. Citrus Processing Short Course Proceedings; University of Florida: Gainesville, 1995; pp 19-39.

(20) Monsoor, M. A.; Kalapathy, U.; Proctor, A. Improved Method for determination of pectin degree of esterification by diffuse reflectance Fourier transform infrared spectroscopy. J. Agric. Food Chem. 2001, 49, 2756-2760.

(21) Heaps, D. A.; Griffiths, P. R. Band shapes in the infrared spectra of thin organic films on metal nanoparticles. Vib. Spectrosc. 2006, 42, $45-50$.

(22) Fishman, M. L.; Walker, P. N.; Chau, H. K.; Hotchkiss, A. T. Flash Extraction of Pectin from Orange Albedo by Steam Injection. Biomacromolecules 2003, 4, 880-889.

(23) Manthey, J. A. Fractionation of Orange Peel Phenols in Ultrafiltered Molasses and Mass Balance Studies of Their Antioxidant Levels. J. Agric. Food Chem. 2004, 52, 7586-7592.

(24) Fukuchi, Y.; Hiramitsu, M.; Okada, M.; Hayashi, S.; Nabeno, Y.; Osawa, T.; Naito, M. Lemon Polyphenols Suppress Diet-induced Obesity by Up-Regulation of mRNA Levels of the Enzymes Involved in $\beta$-Oxidation in Mouse White Adipose Tissue. J. Clin. Biochem. Nutr. 2008, 43, 201-209.

(25) Ortuno, A.; Garcia-Puig, D.; Fuster, M. D.; Perez, M. L.; Sabater, F.; Porras, I.; Garcia-Lidon, A.; Del Rio, J. A. Flavanone and nootkatone levels in different varieties of grapefruit and pummelo. J. Agric. Food Chem. 1995, 43, 1-5.

(26) Ciriminna, R.; Fidalgo, A. M.; Carnaroglio, D.; Tamburino, A.; Cravotto, G.; Ilharco, L. M.; Pagliaro, M. Lemon Essential Oil of Variable Composition by Changing the Conditions of the Extraction from Lemon Peel via Microwave Hydrodiffusion and Gravity. ChemistrySelect 2017, 2, 7123-7127.

(27) Yang, L.; Kruse, B. Revised Kubelka-Munk theory. I. Theory and application. J. Opt. Soc. Am. A 2004, 21, 1933-1941. 